\title{
Características de los sedimentos de la Bahía Oriental del Lago Lácar afectado por la descarga de aguas residuales
}

\author{
Pedro Temporetti ${ }^{\varpi}$; Lidia Antonuk \& Fernando Pedrozo \\ INIBIOMA-CRUB-UNCo, Grupo de Estudios en Calidad de Aguas y Recursos Acuáticos, CONICET-Centro Regional \\ Universitario Bariloche, Universidad Nacional del Comahue, Argentina
}

\begin{abstract}
Resumen. Varios parámetros, físicos y químicos de la columna de agua, son usados para determinar el estado trófico de los ambientes acuáticos. Sin embargo, estos parámetros presentan fluctuaciones estacionales que podrían dificultar la clasificación trófica de un cuerpo de agua. La determinación de los componentes químicos de los sedimentos, complementada con los métodos de fraccionamiento del $\mathrm{P}$, constituyen una herramienta con valor predictivo del estado trófico de los ambientes acuáticos. El objetivo de este trabajo fue evaluar el aporte de las aguas residuales (principalmente fósforo) sobre la calidad de los sedimentos de un lago oligotrófico patagónico (Lago Lácar, San Martín de los Andes, Neuquén), y determinar la influencia sobre la distribución del P en relación con los elementos que controlan su disponibilidad. Los resultados de la química y experiencias de fijación/liberación de P realizadas indican la existencia de una liberación de P desde los sedimentos hacia la columna de agua, fundamentalmente en la zona central de la bahía, lo que origina una carga interna.
\end{abstract}

[Palabras clave: nutrientes, líquidos cloacales]

\begin{abstract}
Aвstract. Sediment characteristics of the East Bay of Lake Lácar affected by sewage discharge: Several physical and chemical parameters of water column are used to determine the trophic status of aquatic environments. However, these parameters show strong seasonal fluctuations that might hinder the trophic classification of a water body. The determination of the chemical components of sediments, supplemented with $\mathrm{P}$ fractionation methods could provide a tool with predictive value of trophic status of aquatic environments. The aim of this study was to evaluate the effect of wastewater (phosphorus primarily) on the quality of the sediments of a Patagonian oligotrophic lake (Lake Lácar, San Martin de Los Andes, Neuquén), and determine the influence on the distribution of $P$ in relation to the elements that control its availability. The chemical results and fixation/release experiences of $\mathrm{P}$ performed, indicate the existence of $\mathrm{P}$ release from the sediments into the water column, mainly in the central area of the bay, causing an internal load.
\end{abstract}

[Keywords: nutrients, sewage]

\section{INTRODUCCIÓN}

Se define eutrofización de un cuerpo de agua como el proceso de adición excesiva de nutrientes inorgánicos [fósforo $(\mathrm{P})$ y nitrógeno $(\mathrm{N})]$ y materia orgánica, que incrementa la producción biológica (Sinke 1992; Wetzel 2001). Los efectos de este proceso sobre los cuerpos de agua son numerosos, tales como la disminución de la transparencia en la columna de agua, el descenso de la concentración de oxígeno disuelto, la aparición de especies algales tóxicas y, en casos muy severos, la mortandad de peces (Wetzel 2001; Golterman 2004). Uno de los nutrientes clave en el proceso de eutrofización es el $\mathrm{P}$, que también puede ser un nutriente limitante en muchos cuerpos de agua (Wetzel 2001; Schindler et al. 2008).

El estado trófico de los ambientes acuáticos se determina a través de parámetros físicos (e.g., transparencia) y químicos (e.g., concentración

\section{Editor asociado: Fernando Unrein}

$\triangle$ pedro.temporetti@crub.uncoma.edu.ar de fósforo total (PT), fósforo reactivo soluble (PRS) y clorofila a (Clo a)) (OECD 1981; Horne \& Goldman 1994; Wetzel 2001; Schindler et al. 2008). Estos parámetros suelen presentar fluctuaciones estacionales que dificultan la clasificación trófica de un cuerpo de agua (Maassen et al. 2005).

Numerosos trabajos relacionan la concentración de PT de la capa superficial de los sedimentos con el de la columna de agua (Håkanson 2003; Carpenter 2005), sobre la base de que el estado trófico está influido por el fenómeno de liberación de P desde los sedimentos. De acuerdo con de Vicente et al. (2003), es esencial conocer la cantidad de P almacenado en los sedimentos, así como las diferentes formas en que se encuentra, dado que puede ser liberado a la columna de agua. Este proceso es citado en la bibliografía como "carga interna" (Böstrom et al 1982; Golterman et al. 1983; Håkanson \& Jansson 1983). 
El contenido de $\mathrm{P}$ en los sedimentos de los lagos depende de la composición química y textural de los sedimentos, de las tasas de sedimentación, de las condiciones físicoquímicas y de los procesos diagenéticos (Anshumali \& Ramanathan 2007; Li et al. 2007). Los factores que influencian el intercambio de $\mathrm{P}$ entre los sedimentos y la columna de agua pueden ser físicos (i.e., temperatura, acción del viento en lagos someros), químicos (i.e., $\mathrm{pH}$, oxígeno disuelto, nitratos, sulfatos) y biológicos (i.e., bioturbación, actividad bacteriana) (Böstrom et al. 1982). El equilibrio entre la fracción del $\mathrm{P}$ asociada a los sedimentos y la disuelta en la columna de agua está determinado por las condiciones redox (ORP) (Böstrom et al. 1982; Sinke 1992; Katsev et al. 2006), los procesos de adsorción, la solubilidad en la fase mineral y la mineralización de la materia orgánica $(\mathrm{MO})$ (Kaiserli et al. 2002).

De acuerdo con Li et al. (2007), la sedimentación de la MO particulada, la reducción del oxígeno disuelto en la superficie del sedimento y la actividad de las bacterias anaeróbicas producen compuestos reductores que provocan una disminución del ORP. Esta disminución provoca la liberación de electrones que pueden ser transferidos a los componentes oxidantes (e.g., óxidos de hierro, aluminio y manganeso). Estos compuestos reducidos se disuelven en la columna de agua, lo que disminuye la capacidad de adsorción del P. Además, los productos de las reacciones de reducción [i.e., aniones orgánicos, amonio $\left(\mathrm{N}-\mathrm{NH}_{4}^{+}\right)$y azufre (S) elemental] compiten con el $\mathrm{P}$ por los sitios de adsorción en el sedimento; esto genera una liberación de fósforo a la columna de agua (Koski-Vähälä et al. 2001).

En relación a los sedimentos, Maassen et al. (2005) mostraron que otros factores pueden afectar el estado trófico de un ambiente acuático. Por ejemplo, la forma de partición del $\mathrm{P}$ en el sedimento afecta las relaciones hierro (Fe):P y aluminio (Al):P, así como a las fracciones asociadas a la materia orgánica y la forma lábil del P. En este sentido, la determinación de los componentes químicos de los sedimentos, complementada con los métodos de fraccionamiento del $\mathrm{P}$, podrían constituir una herramienta con valor predictivo del estado trófico de los ambientes acuáticos (Maassen et al. 2005; Carey \& Rydin 2011).

El objetivo de este trabajo fue evaluar la calidad de los sedimentos (aporte de P) de la
Bahía Oriental del lago Lácar, afectados por la descarga de las aguas residuales. Para cumplir este objetivo se evaluó la física y la química de los sedimentos, se determinaron las fracciones de $\mathrm{P}$ asociadas a los sedimentos, y se estimó su capacidad para retener o liberar fósforo.

\section{MATERIALES Y MÉTODOS}

\section{Área de estudio}

El lago Lácar (Figura 1) se encuentra ubicado en la Cordillera Norpatagónica, al sur de la Provincia de Neuquén, dentro del Parque Nacional Lanín [40¹4' S y 71³0' W (Cordini 1964]. Sobre la margen oriental del lago se encuentra ubicada la ciudad de San Martín de los Andes, cuya población estable es 30000 habitantes. La principal actividad económica de esta ciudad es el turismo. Otras actividades con diferente grado de desarrollo están relacionadas con la industria maderera y ganadera.

Las características morfológicas y químicas del agua del lago se muestran en la Tabla 1. Este lago, de origen glacial y forma alargada, es clasificado térmicamente como monomíctico cálido, ligeramente alcalino, con aguas transparentes, bajo contenido en sales (Temporetti et al. 2008) y del tipo oligotrófico. El cuerpo principal del lago se extiende hacia el oeste conformando el lago Nonthue y el río Hua Hum, por medio del cual desagua hacia el Océano Pacífico. La superficie de la cuenca es de 1048 km²; su población es escasa, salvo en su zona oriental, donde está la ciudad de San Martín de los Andes. La principal bahía del lago es la Bahía Oriental, cuya superficie equivale al 1.2\% del lago, y su volumen es el $0.52 \%$ del lago. Esta bahía presenta una profundidad media de $78.4 \mathrm{~m}$ y características hidrológicas particulares. Está protegida de los vientos predominantes y la temperatura del agua en verano es superior a la del cuerpo principal del lago. El principal afluente del lago es el río Pocahullo, que antes de desembocar en el lago cruza la ciudad de

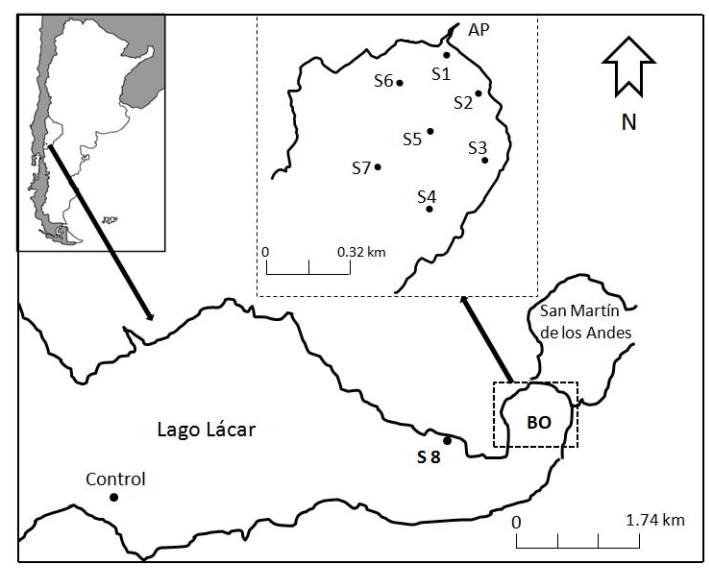

Figura 1. Localización de los sitios de muestreo. BO: Bahía Oriental; AP: río Pocahullo.

Figure 1. Location of sampling sites. BO: East Bay; AP: Pocahullo stream. 
Tabla 1. Características morfológicas, físicas y químicas del lago Lácar.

Table 1. Morphological, physical and chemical characteristics of Lake Lácar.

\begin{tabular}{lc}
\hline Área $\left(\mathrm{km}^{2}\right)$ & 50.3 \\
Volumen $\left(\mathrm{m}^{3}\right)$ & $47 \times 10^{6}$ \\
Z máxima $(\mathrm{m})$ & 277 \\
Z mínima $(\mathrm{m})$ & 166 \\
Tiempo de retención del agua (años) & 5.8 \\
Área de la cuenca $\left(\mathrm{km}^{2}\right)$ & 1048 \\
$\mathrm{pH}$ & 7.72 \\
Conductividad $(\mu \mathrm{S} / \mathrm{cm})$ & 60.2 \\
Oxígeno Disuelto $(\mathrm{mg} / \mathrm{L})$ & 9.61 \\
$\mathrm{PT}(\mu \mathrm{L} / \mathrm{L})$ & 10.3 \\
PRS $(\mu \mathrm{g} / \mathrm{L})$ & $<3$ \\
$\mathrm{NT}(\mu \mathrm{g} / \mathrm{L})$ & 70.2 \\
$\mathrm{~N}-\mathrm{NO}_{3}^{-}+\mathrm{N}-\mathrm{NO}_{2}^{-}(\mu \mathrm{g} / \mathrm{L})$ & 7.6 \\
$\mathrm{~N}^{-\mathrm{NH}_{4}+}(\mu \mathrm{g} / \mathrm{L})$ & $<5$ \\
\hline
\end{tabular}

San Martín de los Andes y recibe aguas de drenaje de las zonas rurales y urbanas de la ciudad.

Hasta el año 1994, la ciudad carecía de una planta de tratamiento de líquidos cloacales, por lo que el río Pocahullo y sus principales tributarios recibían todas las descargas de aguas servidas domiciliarias y rurales. El sistema actual de tratamiento de líquidos cloacales de la ciudad está compuesto por: a) una planta de tratamiento de líquidos cloacales de tipo terciario (construida en 1994 sobre la desembocadura del río Pocahullo, procesa los efluentes del $60 \%$ de la población); b) una planta con tanques de aireación y sedimentación (ubicada al este de la ciudad y aguas arriba del río Pocahullo); y c) lagunas de estabilización (aguas arriba de la planta anterior). Los efluentes de las dos últimas plantas son conducidos a cursos de agua que forman parte de la cuenca del río Pocahullo, lo que incrementa las concentraciones de nutrientes en las zonas de descarga (Cooke et al. 2006; Temporetti et al. 2007, 2008). Temporetti et al. (2008) informaron que la concentración de nutrientes en el Pocahullo había aumentado entre 8.3 y 23 veces en el casco urbano respecto del sitio control del río. Por ejemplo, el PT fue en el sitio control de $31 \mu \mathrm{g} / \mathrm{L}$ y de $260 \mu \mathrm{g} / \mathrm{L}$, en promedio, para los 5 sitios ubicados dentro del casco urbano. Por otro lado, estos autores también encontraron, para igual periodo de estudio, que las concentraciones de nutrientes totales y disueltos incrementaron entre 1.3 y 1.7 veces en el sitio de muestreo del lago Lácar que recibe la descarga del río Pocahullo, respecto del sitio control en el lago. Por ejemplo, el PT en el sitio del lago donde descarga el río Pocahullo fue de $10.2 \mu \mathrm{g} / \mathrm{L}$, mientras que la concentración de fósforo en el sitio control del lago fue $6.0 \mu \mathrm{g} / \mathrm{L}$.

El muestreo de sedimentos se realizó en febrero de 2008. Se establecieron 9 sitios de muestreo (Figura 1). Siete sitios estuvieron distribuidos dentro de la Bahía Oriental, uno sobre la margen norte del lago y fuera de la bahía, y un sitio control a $20 \mathrm{~km}$ de San Martín de Los Andes. La elección de este último se realizó según las características físico-químicas del agua del lago en ese punto, establecidas a partir de trabajos previos realizados por Temporetti et al. $(2000,2006)$.

\section{A campo}

Las muestras de sedimento fueron tomadas con una draga tipo Ekman-Birge. Las profundidades de extracción figuran en la Tabla 2. El pH y el potencial redox de cada muestra se midieron "in situ" con un medidor de $\mathrm{pH}$ Orion 256 equipado con electrodos específicos y corrección por temperatura $\left(25^{\circ} \mathrm{C}\right)$. Las muestras fueron almacenadas en bolsas plásticas y conservadas en frío y oscuridad hasta su análisis en laboratorio según las recomendaciones de APHA (1995).

\section{En el laboratorio}

1) Procesamiento de las muestras. Todas las muestras de sedimento fueron secadas en estufa a $60{ }^{\circ} \mathrm{C}$, homogeneizadas en mortero cerámico y tamizadas a través de una malla de $500 \mu \mathrm{m}$ (Newark, ASTM N 36 USA Standard Series Sieves) para eliminar la fracción gruesa menos reactiva. Los análisis de composición textural, química, nutrientes y fracciones de $\mathrm{P}$ se realizaron sobre todas las muestras extraídas (sitios S1, S2, S3, S4, S5, S6, S7, S8 y sitio Control) (Figura 1). Para los análisis mineralógicos y las experiencias de fijación/liberación de $\mathrm{P}$ en los sedimentos se seleccionaron los siguientes sitios según la concentración de PT determinada en este estudio: S1 (frente a la desembocadura del río Pocahullo), S7 (en el centro de la bahía y con una concentración de fósforo total (PT) elevada respecto del resto de los sitios) y el sitio control (Figura 1).

2) Métodos analíticos. Una fracción del sedimento seco y tamizado se destinó para el análisis granulométrico, realizado con un equipo Mastersizer 2000 optical unit, Hydro 2000 MV en el laboratorio de química del Instituto Argentino de Oceanografía (IADO) de Bahía Blanca. Se determinaron las siguientes fracciones texturales de acuerdo a la escala Wentworth, modificada por Friedman et al. (1992): arcilla $(<3 \mu \mathrm{m})$, limo medio

Tabla 2. Profundidades de extracción de las muestras, $\mathrm{pH}$ y ORP medidos en las muestras de sedimentos.

Table 2. Extraction depths samples, $\mathrm{pH}$ and ORP measured in sediment samples.

\begin{tabular}{cccc}
\hline Sitio & $\begin{array}{c}\text { Profundidad de } \\
\text { extracción }(\mathrm{m})\end{array}$ & $\mathrm{pH}$ & ORP $(\mathrm{mV})$ \\
\hline Control & 45 & 6.50 & +138.4 \\
S1 & 17 & 6.45 & +107.3 \\
S2 & 19 & 6.58 & +124.5 \\
S3 & 19 & 6.52 & +96.8 \\
S4 & 105 & 6.66 & +81.1 \\
S5 & 36 & 6.67 & +63.6 \\
S6 & 40 & 6.36 & +53.4 \\
S7 & 95 & 6.46 & +58.4 \\
S8 & 125 & 6.55 & +66.4 \\
\hline
\end{tabular}


fino (3.9 a $3.1 \mu \mathrm{m})$, limo fino $(7.8$ a $3.8 \mu \mathrm{m})$, limo medio (15.6 a $7.7 \mu \mathrm{m})$, limo grueso (31.2 a $15.5 \mu \mathrm{m})$, arena muy fina $(62.5 \mathrm{a} 31.1 \mu \mathrm{m})$, arena fina $(125 \mathrm{a}$ $62.4 \mu \mathrm{m})$, arena media $(250 \mathrm{a} 124 \mu \mathrm{m})$ y arena gruesa (500 a $249 \mu \mathrm{m})$.

Analizamos fósforo Total (PT) de acuerdo con Carter (1993). Una sub muestra de sedimento fue digerida a $440{ }^{\circ} \mathrm{C}$ con $\mathrm{SO}_{4} \mathrm{H}_{2}$ concentrado y peróxido de hidrógeno $\left(\mathrm{H}_{2} \mathrm{O}_{2}\right)$ al $30 \%$. Luego de la digestión se determinó el contenido de PT de acuerdo a la metodología propuesta por Murphy \& Riley (1962). Las concentraciones de nitrógeno total (NT) y carbono total (CT) se determinaron mediante un analizador automático Thermo FlashEA 1112. La caracterización química de los sedimentos (expresada como óxidos de los elementos) se realizó mediante el análisis SEM-EDAX y la mineralogía por difracción de Rx (Phillips X Ray Generator PW 3719). Determinamos las diferentes fracciones de $\mathrm{P}$ de acuerdo con el esquema de fraccionamiento propuesto por Hieltjes \& Lijklema (1980). Se discriminó la fracción lábil (P-lábil), extraída con $\mathrm{ClNH}_{4} 1 \mathrm{M}$, la fracción unida al Fe y al Al (P-Fe/ $\mathrm{Al}$ ), extraída con $\mathrm{NaOH} 0.1 \mathrm{M}$, y la fracción unida al calcio $(\mathrm{Ca})(\mathrm{P}-\mathrm{Ca})$, extraída con $\mathrm{HCl} 0.5 \mathrm{M}$. La fracción de $\mathrm{P}$ unida a la $\mathrm{MO}$ (P-MO) se calculó de la diferencia entre el $\mathrm{PT}$ y de la suma de las fracciones anteriores (P-MO=PT- $\Sigma$ P-Labil+P-Fe $/ \mathrm{Al}+\mathrm{P}-\mathrm{Ca})$.

Las experiencias de fijación/liberación de $\mathrm{P}$ se realizaron incubando $0.2 \mathrm{~g}$ de sedimento seco con $10 \mathrm{ml}$ de soluciones estándares de diferentes concentraciones de $\mathrm{P}(0,1700,2700,3700,4500$, 5500, 8500 y $10000 \mu \mathrm{g}$ P/L, preparadas a partir de una solución estándar de fosfatos Merck 1.19898 .0500 de $1000 \mathrm{mgP} / \mathrm{L}$ ) durante $48 \mathrm{~h}$ en oscuridad y a temperatura ambiente $\left(20^{\circ} \mathrm{C}\right)$. Luego se determinó el PRS en solución (Murphy \& Riley 1962). Los resultados fueron analizados y graficados en función de las ecuaciones de a) Freundlich (Langmuir 1997; Golterman 2004):

Po + Pads $=K^{*}(\text { Pdis })^{b}$

En esta ecuación,

Po: concentración inicial de fósforo en el sedimento

Pads: fósforo fijado al sedimento

Pdis: concentración de fósforo remanente en solución

$\mathrm{K}$ : constante

b: constante (varía entre 0 y 1 )

b) Langmuir (Langmuir 1997; Golterman 2004):

Pdis $/[$ Po+Pads $]=1 /\left(k^{*}\right.$ Pmáx $)+\left[(1 /(\text { Pmáx }))^{*}\right.$ Pdis $]$

En esta ecuación,

Pmáx: capacidad de fijación máxima de $\mathrm{P}$ por parte del sedimento

k: constante que vincula a la energía de unión del P con el sedimento (Olsen \& Watanabe 1957)
Todos los análisis químicos fueron realizados por triplicado y las concentraciones expresadas como peso seco (p.s.) de las muestras.

3) Análisis estadístico de los datos. Se realizaron dos análisis de Componentes Principales (ACP) para estudiar la relación entre las variables y los sitios. El primer análisis incluyó las variables texturales y el segundo las variables químicas. La matriz de observaciones para el primer ACP tuvo diez columnas que representaron a las variables texturales medidas (arcilla, limo medio fino, limo fino, limo medio, limo grueso, arena media fina, arena fina, arena media, arena gruesa y arena media gruesa), y nueve filas que representaron a los sitios de muestreo. Para el segundo ACP, la matriz de observaciones estuvo formada por once columnas que representaron a las variables medidas $\left(\mathrm{SiO}_{2}\right.$ $\mathrm{Al}_{2} \mathrm{O}_{3}, \mathrm{Fe}_{2} \mathrm{O}_{3}, \mathrm{CaO}, \mathrm{P}$-lábil, P-Fe/Al, P-Ca, P-Org, $\mathrm{PT}$, NT y CT), y nueve filas que representaron a todos los sitios de muestreo. Todas las variables se consideraron activas en ambos análisis.

\section{Resultados}

El pH para todos los sitios resultó ligeramente ácido (promedio de 6.53), con valores comprendidos entre 6.36 (sitio S6) y 6.67 (sitio S5) (Tabla 2). El ORP resultó positivo pero bajo en todos los sitios; su valor osciló entre $+53.4 \mathrm{mV}$ (sitio S8) y $+138.0 \mathrm{mV}$ (sitio control) (Tabla 2).

En todas las muestras predominó la fracción media gruesa (entre $15.6 \mu \mathrm{m}$ y $500 \mu \mathrm{m}$ ) (limo grueso a arena media gruesa), que varió entre $16.7 \%$ (sitio S2) y $18.5 \%$ (sitio S1). La fracción de arena muy gruesa fue baja (2.35 $\%$ en promedio). Las fracciones finas $(<3 \mu \mathrm{m}$ y $15.63 \mu \mathrm{m})$ resultaron bajas, variando, en promedio, entre $1.1 \%$ (sitio S1) y $4.3 \%$ (sitio S8) (Tabla 3).

La concentración de PT en el sitio control fue $636 \mu \mathrm{gP} / \mathrm{g}$ p.s., 1.7 veces más baja que en los sitios costeros (en promedio, $1116 \mu \mathrm{gP} / \mathrm{g}$ p.s. $\pm 168 \mu \mathrm{gP} / \mathrm{g})$ y 2.5 veces más baja que los sitios profundos (en promedio, $1585 \mu \mathrm{gP} / \mathrm{g}$ p.s. $\pm 112 \mu \mathrm{gP} / \mathrm{g}$ ). Dentro de la Bahía Oriental, las menores concentraciones se registraron en los sitios costeros ( $\mathrm{S} 1=1017 \mu \mathrm{gP} / \mathrm{g}$ p.s., S2 $=1310 \mu \mathrm{gP} / \mathrm{g}$ p.s. y $\mathrm{S} 3=1022 \mu \mathrm{gP} / \mathrm{g}$ p.s.), mientras que las mayores concentraciones se observaron en los sitios más profundos y centrales de la Bahía: S4 (1630 $\mu \mathrm{gP} / \mathrm{g}$ p.s.), S6 (1674 $\mu \mathrm{gP} / \mathrm{g}$ p.s.) y S7 (1640 $\mu \mathrm{gP} / \mathrm{g}$ p.s.).

La concentración media de NT para todos los sitios fue $39.8 \mathrm{mgN} / \mathrm{g}$ p.s., con valores comprendidos entre 15.0 y $56.0 \mathrm{mgN} / \mathrm{g}$ p.s. en los sitios S1 y S7, respectivamente (Tabla 4). Por otro lado, las concentraciones de CT resultaron 
Tabla 3. Composición textural de los sedimentos. Ar: arcilla; Lmf: limo medio fino; Lf: limo fino; Lm: limo medio; Lg: limo grueso; Amf: arena media fina; Af: arena fina; Am: arena media; Ag: arena gruesa y Amg: arena media gruesa.

Table 3. Textural composition of the sediments. Ar: clay; Lmf: silt half fine; Lf: silt fine; Lm: silt medium; Lg: silt thick; Amf: sand medium fine; Af: fine sand; Am: sand medium; Amg: sand half gravel and Ag: sand thick.

\begin{tabular}{|c|c|c|c|c|c|c|c|c|c|c|}
\hline & $\begin{array}{c}\text { Ar } \\
<3.0 \mu \mathrm{m} \\
(\%)\end{array}$ & $\begin{array}{c}\text { Lmf } \\
3.0-3.9 \\
\mu \mathrm{m} \\
(\%)\end{array}$ & $\begin{array}{c}\text { Lf } \\
3.9-7.8 \\
\mu \mathrm{m} \\
(\%)\end{array}$ & $\begin{array}{c}\mathrm{Lm} \\
7.8-15.6 \\
\mu \mathrm{m} \\
(\%)\end{array}$ & $\begin{array}{c}\mathrm{Lg} \\
15.6-31.2 \\
\mu \mathrm{m} \\
(\%)\end{array}$ & $\begin{array}{c}\text { Amf } \\
31.2-62.5 \\
\mu m \\
(\%)\end{array}$ & $\begin{array}{c}\text { Af } \\
62.5-125 \\
\mu \mathrm{m} \\
(\%)\end{array}$ & $\begin{array}{c}\text { Am } \\
125-250 \\
\mu m \\
(\%)\end{array}$ & $\begin{array}{c}\mathrm{Ag} \\
249-500 \\
\mu \mathrm{m} \\
(\%)\end{array}$ & $\begin{array}{c}\text { Amg } \\
500-1000 \\
\mu_{(\%)} \\
(\%)\end{array}$ \\
\hline Control & 1.42 & 0.48 & 1.94 & 4.83 & 9.79 & 16.43 & 25.45 & 25.26 & 13.04 & 1.36 \\
\hline S 1 & 0.34 & 0.27 & 1.15 & 2.74 & 5.37 & 11.36 & 24.90 & 31.80 & 18.88 & 3.19 \\
\hline $\mathrm{S} 2$ & 2.38 & 0.82 & 3.36 & 6.61 & 11.50 & 17.70 & 22.84 & 18.93 & 12.49 & 3.37 \\
\hline S 3 & 1.09 & 0.40 & 1.68 & 4.31 & 7.66 & 15.40 & 29.58 & 25.50 & 11.27 & 3.11 \\
\hline S 4 & 2.06 & 0.61 & 2.69 & 7.03 & 14.44 & 21.96 & 24.59 & 16.97 & 8.19 & 1.46 \\
\hline S 5 & 1.77 & 0.51 & 2.24 & 5.86 & 13.26 & 23.26 & 26.09 & 17.16 & 8.38 & 1.47 \\
\hline S 6 & 1.73 & 0.54 & 2.49 & 6.57 & 13.15 & 19.83 & 23.66 & 18.50 & 10.68 & 2.85 \\
\hline S 7 & 1.95 & 0.57 & 2.59 & 6.81 & 13.42 & 20.23 & 23.80 & 18.16 & 9.97 & 2.50 \\
\hline S 8 & 2.95 & 0.94 & 3.75 & 9.59 & 17.00 & 19.81 & 18.92 & 25.23 & 9.39 & 1.85 \\
\hline
\end{tabular}

bajas (en promedio, $2.9 \mathrm{mgC} / \mathrm{g}$ p.s. para todos los sitios). Al igual que lo observado con el NT, el sitio $S 1$ mostró la concentración más baja de $\mathrm{CT}(1.0 \mathrm{mgC} / \mathrm{g}$ p.s.), mientras que los sitios $\mathrm{S} 4$ y $\mathrm{S7}$ presentaron las concentraciones más altas (4.0 mgC/g p.s.) (Tabla 4).

La Tabla 4 muestra la composición química, expresada como óxidos de cada elemento. La relación de elementos fue: $\mathrm{Si}>>\mathrm{Al}>>\mathrm{Fe}>\mathrm{Ca} \geq \mathrm{Na}>\mathrm{K} \geq \mathrm{Ti}$. En todos los casos, el $\mathrm{SiO}_{2}$ fue, en promedio, el óxido más importante (61.8\%). Los óxidos $\mathrm{Al}_{2} \mathrm{O}_{3}$ y $\mathrm{Fe}_{2} \mathrm{O}_{3}$ siguieron al $\mathrm{SiO}_{2}$ en importancia $(19.6 \%$ y $8.2 \%$ en promedio, respectivamente). El análisis mineralógico realizado en los sitios S1, S7 y control permitió establecer la presencia de cuarzo $\left(\mathrm{SiO}_{2}\right)$, mineral estable y mayoritario en las arcillas. Por otro lado, se determinó la existencia de: albita $\left(\mathrm{Na}\left(\mathrm{AlSi}_{3} \mathrm{O}_{8}\right)\right)$ en los sitios $\mathrm{S} 1$ y S7 y anortita $\left(\mathrm{Na}\left(\mathrm{Al}_{2} \mathrm{Si}_{2} \mathrm{O}_{8}\right)\right)_{8}$ en los tres sitios.

La fracción principal en todos los sitios fue la unida a la materia orgánica (P-MO, 63.7\% en promedio) (Figura 2). La fracción de P ligada a óxidos e hidróxidos de $\mathrm{Fe}$ y $\mathrm{Al}$ ( $\mathrm{P}-\mathrm{Fe} / \mathrm{Al}$ ) fue la segunda fracción en importancia; representó, en promedio, el $23.1 \%$. La fracción del P ligada a óxidos de $\mathrm{Ca}(\mathrm{P}-\mathrm{Ca})$ fue, en promedio, $12.6 \%$, mientras que la fracción de P lábil (P-Lábil), fue muy baja en todos los sitios (en promedio, $0.6 \%)$.

En los tres sitios analizados, los resultados se ajustaron tanto a las isotermas de Freundlich (Figura 3A) $\left(R^{2}=0.85, P<0.01\right.$ para el sitio control; $R^{2}=0.89, P<0.01$ para el sitio S1, y $R^{2}=0.88, P<0.01$ para el sitio $\mathrm{S} 7$, respectivamente) como a las de Langmuir (Figura 3B) $\left(R^{2}=0.99, P<0.01\right.$ para los tres sitios). A partir de esta última ecuación se estimó la capacidad máxima de fijación de $\mathrm{P}$ al sedimento, que para el sitio control fue 930 $\mathrm{\mu g} / \mathrm{g}, 1300 \mu \mathrm{g} / \mathrm{g}$ para el sitio S1 y $1950 \mu \mathrm{g} / \mathrm{g}$ para el sitio S7. Las constantes calculadas de unión del $\mathrm{P}$ al sedimento (Olsen \& Watanabe 1957) resultaron $0.009,0.020$ y 0.026 para los sitios control, S1 y S7, respectivamente.

Tabla 4. Composición química de los sedimentos. PT: fósforo total; NT: nitrógeno total y CT: carbono total.

Table 4. Chemical composition of the sediments. TP: total phosphorus; TN: total nitrogen and TC: total carbon.

\begin{tabular}{|c|c|c|c|c|c|c|c|c|c|c|c|}
\hline & \multicolumn{4}{|c|}{ Sitios costeros $=$ texturas gruesas } & \multirow[b]{2}{*}{ Control } & \multicolumn{6}{|c|}{ Sitios profundos $=$ texturas finas } \\
\hline & S1 & S2 & S3 & Promedio & & Promedio & S4 & S5 & S6 & S7 & S8 \\
\hline PT ( $\mu$ g/g p.s.) & 1017 & 1310 & 1022 & 1116 & 636 & 1578 & 1630 & 1391 & 1674 & 1640 & 1588 \\
\hline NT (mg/g p.s.) & 15.0 & 41.0 & 33.0 & 30.0 & 38.0 & 48.2 & 49.0 & 39.0 & 50.0 & 56.0 & 38.0 \\
\hline CT (mg/g p.s.) & 1.0 & 3.0 & 2.0 & 2.0 & 3.0 & 3.0 & 4.0 & 3.0 & 3.0 & 4.0 & 3.0 \\
\hline $\mathrm{SiO}_{2}(\%)$ & 57.0 & 61.1 & 61.2 & 59.8 & 61.1 & 63.0 & 64.0 & 60.9 & 64.1 & 61.0 & 65.6 \\
\hline $\mathrm{Al}_{2} \mathrm{O}_{3}(\%)$ & 22.5 & 19.0 & 20.0 & 20.4 & 19.0 & 19.2 & 19.4 & 20.2 & 17.6 & 20.4 & 18.1 \\
\hline $\mathrm{Fe}_{2} \mathrm{O}_{3}(\%)$ & 9.0 & 9.1 & 8.0 & 8.7 & 9.6 & 7.8 & 6.6 & 7.8 & 8.9 & 8.3 & 7.0 \\
\hline $\mathrm{CaO}(\%)$ & 3.7 & 3.7 & 3.4 & 3.6 & 2.8 & 2.8 & 2.8 & 3.1 & 2.6 & 3.2 & 2.6 \\
\hline $\mathrm{Na}_{2} \mathrm{O}(\%)$ & 3.4 & 2.8 & 3.3 & 3.2 & 2.8 & 3.0 & 3.5 & 3.6 & 2.5 & 3.0 & 2.4 \\
\hline $\mathrm{K}_{2} \mathrm{O}(\%)$ & 1.1 & 1.0 & 1.2 & 1.1 & 1.3 & 1.1 & 1.1 & 1.2 & 1.1 & 1.0 & 1.2 \\
\hline $\mathrm{TiO}_{2}(\%)$ & 0.8 & 0.9 & 0.9 & 0.9 & 1.0 & 0.9 & 0.8 & 0.9 & 0.9 & 0.9 & 0.9 \\
\hline
\end{tabular}




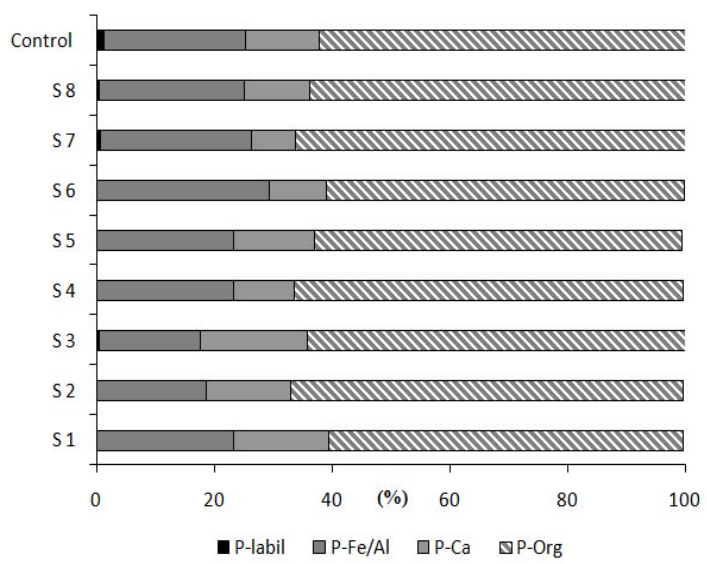

Figura 2. Fraccionamiento del P en los sedimentos. P-lábil: fósforo lábil; $\mathrm{P}-\mathrm{Fe} / \mathrm{Al}$ : fósforo ligado a óxidos e hidróxidos de hierro y aluminio; P-Ca: fósforo ligado a compuestos de calcio, y P-Org: fósforo ligado a la materia orgánica.

Figure 2. Fractionation of $\mathrm{P}$ in sediments. P-labile: phosphorus labile; P-Fe/Al: phosphorus bound to oxides and hydroxides of iron and aluminum; P-Ca: phosphorus bound to calcium compounds, and P-Org: phosphorus bound to organic matter.

\section{Análisis estadístico}

El primer plano factorial de los sitios (Figura 4A) mostró que los que más contribuyeron al primer eje fueron el sitio S1 (en el semieje positivo) y el S8 (en el negativo), mientras que para el segundo eje correspondió a los sitos S2, S5 y S8. Todos los sitos tuvieron una alta representación en el primer plano factorial, con excepción del sitio control. Por otro lado, si se consideran las variables analizadas (Figura 4B), los dos primeros ejes explicaron $91.5 \%$ de la variabilidad de los datos. Las variables que más contribuyeron a la conformación del primer eje fueron arcilla, limo muy fino, fino, medio y grueso y arena muy fina. La arena media también contribuyó al primer eje, pero presentó una correlación negativa con el grupo anterior. Las fracciones arena fina y muy gruesa fueron explicadas por ambos ejes. En este análisis se pueden distinguir dos grupos (Figura 4A). El Grupo I, formado por los sitios control, S1, S2 y S3 se caracterizó por porcentajes altos de arena media y bajas proporciones de arena media fina y limo grueso (Figura 4B). Este grupo asocia los sitios más cercanos a la costa; las profundidades de extracción de las muestras variaron entre 17 y $45 \mathrm{~m}$. El Grupo II estuvo integrado por los sitios S4, S5, S6, S7 y S8; se caracterizó por porcentajes elevados de limo grueso y arena muy fina, y bajas proporciones de arena media (Figura 4B). Las profundidades de extracción de las muestras en estos sitios variaron entre 40 y $125 \mathrm{~m}$.
La Figura 5 (A y B), muestra los resultados del ACP realizado con el análisis químico. Los dos primeros ejes del ACP explicaron $67.4 \%$ de la variabilidad de los datos (Figura 5A). Los sitios que más contribuyeron al primer eje fueron los sitios S1 y S3 (en el semieje positivo), y los sitios S4 y S8 en el eje negativo. En relación al segundo eje, los sitos S2, S3 y control contribuyeron de manera importante en el semieje positivo, mientras que el sitio S6 fue el que mas contribuyó en el semieje negativo. La calidad de representación del sito S5 fue inferior a la calidad del resto de los sitios. Por otro lado, las variables (Figura 5B) que mas contribuyeron a la conformación del primer eje fueron $\mathrm{P}-\mathrm{Ca}, \mathrm{CaO}$ y $\mathrm{Al}_{2} \mathrm{O}_{3}$ (en el semieje positivo) y CT, PT y NT y $\mathrm{SiO}_{2}$ (en el semieje negativo). Se observó que las variables $\mathrm{P}-\mathrm{Ca}$ y $\mathrm{CaO}$ estuvieron asociadas entre sí, al igual que el CT, el PT y el NT. El segundo eje estuvo conformado por P-Org, $\mathrm{Fe}_{2} \mathrm{O}_{3}$ y P-Fe/Al. Las once variables estuvieron bien representadas en los dos primeros ejes factoriales. En este análisis identificamos 4 grupos: el Grupo I (formado sólo por el sitio S1, con bajas concentraciones de nutrientes totales y de $\mathrm{SiO}_{2}$ y $\mathrm{Al}_{2} \mathrm{O}_{3}$ ), el Grupo II (formado por los sitios $\mathrm{S}_{2}^{2}$ y S3 y caracterizado por porcentajes bajos de P$\mathrm{Fe} / \mathrm{Al}$ ), el Grupo III (integrado sólo por el sitio
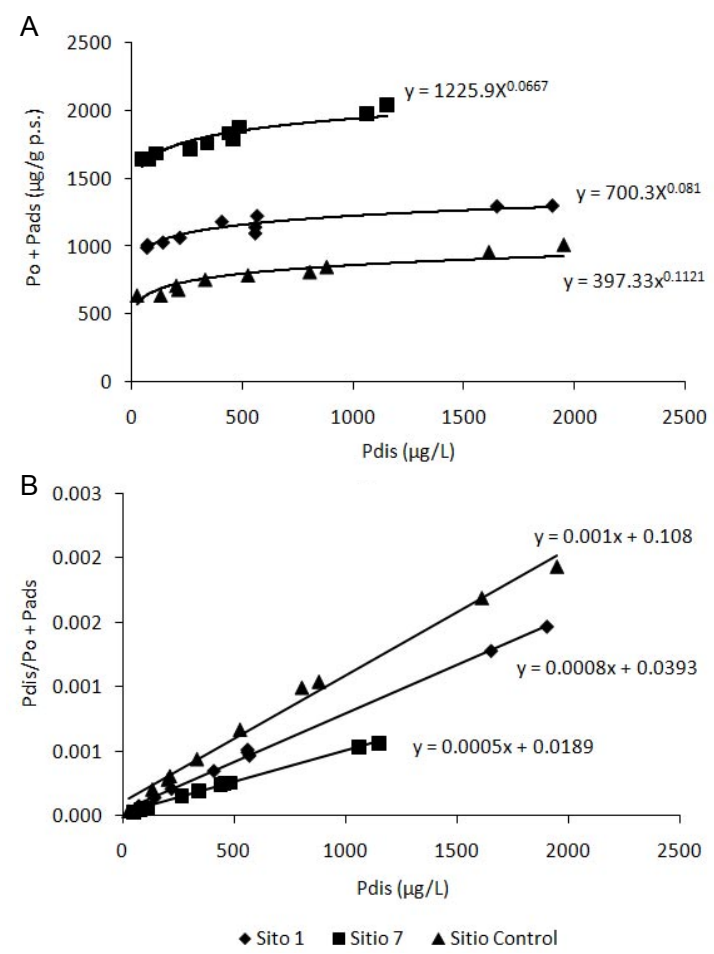

Figura 3. Isotermas de adsorción de Freundlich (A) y Langmuir (B).

Figure 3. Freundlich (A) and Langmuir (B) adsorption isotherms. 


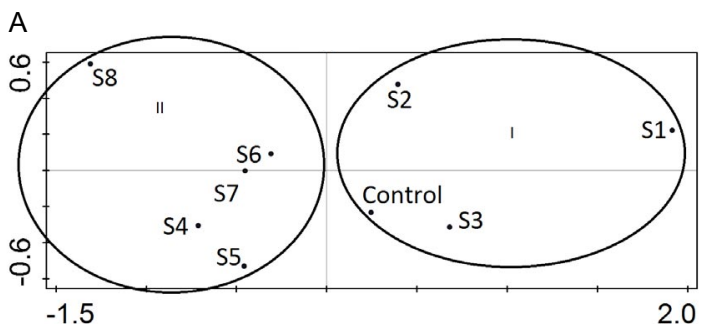

B

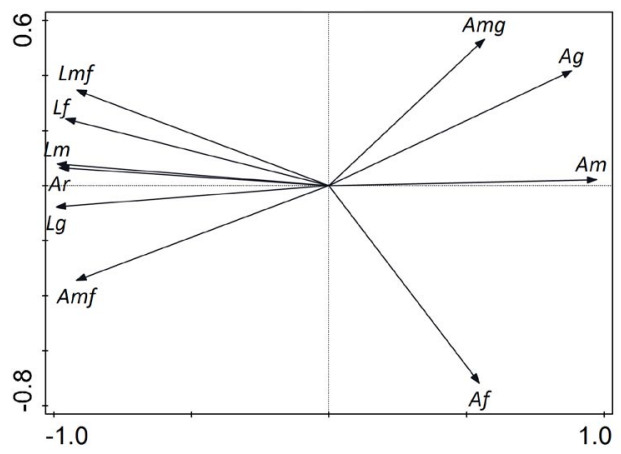

Figura 4. Primer plano tactorial donde se observan la distribución de los individuos (A) y las variables (B) para el análisis textural de las muestras de sedimentos.

Figure 4. First factorial plane where we see the distribution of individuals (A) and variables (B) for textural analysis of sediment samples.

control y caracterizado por porcentajes altos de P-lábil y $\mathrm{Fe}_{2} \mathrm{O}_{3}$ y bajo P-Org). Por último, el Grupo IV, integrado por los sitios S4, S7, S8 y S6, y caracterizado por elevados porcentajes de PT, CT, NT, $\mathrm{SiO}_{2}$ P-Fe/Al y P-Org, y najos porcentajes de $\mathrm{P}-\mathrm{Ca}, \mathrm{CaO}$ y $\mathrm{Al}_{2} \mathrm{O}_{3}$.

\section{DisCUSIÓN}

La diversidad de minerales en los sedimentos lacustres se debe a la influencia significativa de la geología de la cuenca y a la composición del suelo (Last 2001b). Los minerales encontrados en los sedimentos analizados pertenecen al grupo de los feldespatos y se presentan en la mayoría de las rocas eruptivas. Además, en el sitio control encontramos silicatos de $\mathrm{Na}$ y $\mathrm{Mg}$. De acuerdo a estos resultados, el origen de los sedimentos es alóctono (i.e., material detrítico transportado al lago a través de las corrientes de agua, erosión de las costas y escorrentía superficial).

De acuerdo con Last (2001a), los mecanismos y las tasas de transferencia de sustancias entre el agua intersticial, los sedimentos y el agua de la interfase agua-sedimento están controlados, entre otros factores, por la textura fina (de mayor reactividad). Los sedimentos analizados en este trabajo estuvieron dominados por las texturas medias a medias-gruesas (desde 7.8 a $500 \mu \mathrm{m}$ ), lo cual presupone una menor capacidad de retención de nutrientes, en especial fósforo. El ACP diferenció a los sitios de mayor cercanía a la costa, dominados por las fracciones texturales más gruesas, respecto a aquellos localizados en la parte central de la Bahía Oriental, en los que prevalecieron las fracciones de texturas medias. Tanto las características mineralógicas como las texturales se hallan en relación con el bajo contenido de nutrientes de los sedimentos del sitio controllos cuales denotan su origen en la roca andesítica.

Los sedimentos colectados en todos los sitios dentro de la Bahía Oriental presentaron concentraciones de $\mathrm{P}$ mucho más elevadas que las medidas en el sedimento del sitio control. Las concentraciones de PT medidas en el sedimento del sitio control fueron similares a las de los sedimentos de ambientes lacustres andino-patagónicos no afectados por actividades antrópicas, tales como el Lago Argentino (PT $=693 \mu \mathrm{g} / \mathrm{g}$ ), el Lago Pueyrredón $(\mathrm{PT}=735 \mu \mathrm{g} / \mathrm{g}$ ) y el Lago Cardiel $(\mathrm{PT}=714 \mu \mathrm{g} /$ g) (Temporetti et al. 2014).

A

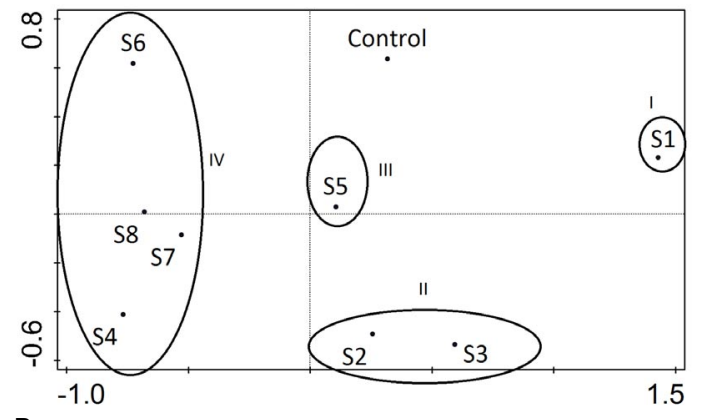

B

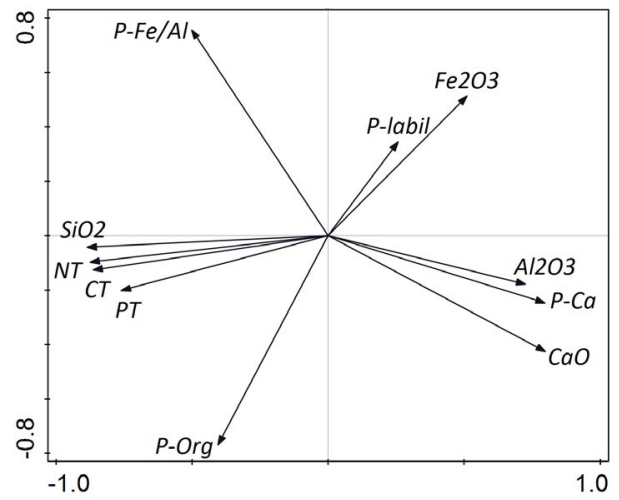

Figura 5. Primer Plano Factorial donde se observan la distribución de los individuos (A) y las variables (B) para el análisis químico de las muestras de sedimentos.

Figure 5. First factorial plane where we see the distribution of individuals (A) and variables (B) for the chemical analysis of sediment samples. 
Los resultados muestran que los sedimentos de la bahía se han enriquecido en $\mathrm{P}$, aportado por el río Pocahullo. Se observó una tendencia a incrementar las concentraciones de $\mathrm{P}$ en los sitios ubicados sobre la margen este y centro de la bahía, donde el Pocahullo reduce su velocidad de corrientey deposita en su extremo más distal la fracción más fina de sedimento debido a su menor velocidad de sedimentación. Por otro lado, si bien no existe un estudio de corrientes del lago, la concentración elevada de $\mathrm{P}$ en el sedimento del sitio S8 podría indicar que la exportación de fósforo desde la Bahía Oriental hasta el cuerpo principal del lago sería hacia su margen norte.

Las concentraciones de $\mathrm{N}$ en los sedimentos muestran un patrón similar al encontrado con el $\mathrm{P}$, aunque no hubo una marcada diferencia entre las concentraciones medidas entre el sitio control y el resto de los sitios. El sitio $\mathrm{S} 1$ presentó la concentración de $\mathrm{N}$ más baja respecto del resto de los sitios. El $\mathrm{N}$ en forma particulada está en su mayoría ligado a estructuras orgánicas. De acuerdo con Golterman (2004), las concentraciones de N en el sedimento oscilan entre 0.05 y $0.5 \%$ (0.5-5 $\mathrm{mg} \mathrm{N} / \mathrm{g}$ ). Valores más altos se reflejarían en un incremento de la biomasa de la comunidad fitoplanctónica. En todos los sitos muestreados en este trabajo, el porcentaje de $\mathrm{N}$ resultó un orden de magnitud más elevado que el postulado por Golterman (2004). Aunque la explicación puede vincularse también al tipo de lago y al aporte alóctono de materia orgánica desde su entorno, cabe considerar que el lago Lácar experimentó un incremento importante (entre 1.8 y 4.5 veces mayor en los muestreos realizados en verano respecto de los realizados en otoño e invierno) de la densidad fitoplanctónica en el mismo periodo de trabajo (Temporetti et al. 2008).

El análisis estadístico mostró que los sitios menos profundos presentaron concentraciones más bajas de nutrientes y mayores porcentajes de P-lábil, $\mathrm{Fe}_{2} \mathrm{O}_{3}$ y $\mathrm{Al}_{2} \mathrm{O}_{3}$ respecto de los sitios más profundos. Por el contrario, los sitios profundos presentaron concentraciones mayores de nutrientes (PT, NT, CT) respecto del sitio control y de los sitios costeros (ricos en $\mathrm{SiO}_{2}$ y fracciones de $\mathrm{P}$ ligadas a oxi-hidroxidos de hierro y aluminio y a la materia orgánica).

El fraccionamiento del $\mathrm{P}$ indicó que el mecanismo que controla este nutriente, en los sedimentos sería la materia orgánica conjuntamente con los oxi-hidróxidos de hierro y aluminio. Estos resultados guardan relación con la composición química de los sedimentos, en donde los porcentajes de los óxidos de aluminio y hierro fueron los más elevados en todos los sitios después de los óxidos de sílice. Este mecanismo de control, característico de los sedimentos ligeramente ácidos a neutros (Golterman 2004), también ha sido observado en ambientes similares de la región Andino-Patagónica (Temporetti 1998; Temporetti \& Pedrozo 2000).

Los resultados de los estudios de fijación de $P$ indicaron que los sedimentos analizados poseen una baja capacidad de fijación en los tres sitios analizados, si se tiene en cuenta la concentración de $\mathrm{P}$ de estos sedimentos. El sitio control presentó $46 \%$ de capacidad de fijación; el S1, 30\%, y el S13, 16\%. Estos resultados se relacionan con el mayor contenido de arcillas de los sedimentos costeros, en especial el sitio S2, enriquecido en la fracción fina por aporte del Pocahullo. La mineralización de la materia orgánica produce cambios tanto en el pH como en el ORP (Wang et al. 2008). La reducción del oxígeno disuelto en la superficie de los sedimentos y la actividad microbiana anaeróbica en los mismos resulta en la producción de compuestos reductores que descienden el ORP de los sedimentos (Li et al. 2007). Un modelo difundido en limnología es el de Einsele $(1936,1938)$ y Mortimer (1941, 1942). Estos autores demostraron que en anaerobiosis se libera más $\mathrm{P}$ que en condiciones aeróbicas debido a la liberación del P ligado al hierro cuando existe una reducción de ión férrico $\left(\mathrm{Fe}^{3+}\right)$ a ferroso $\left(\mathrm{Fe}^{2+}\right)$ en la superficie del sedimento a potenciales redox por debajo de $+200 \mathrm{mV}$ (Golterman 2004). En este sentido, los ORP registrados en los sedimentos de la Bahía Oriental resultaron inferiores al valor mencionado (variaron entre $+53 \mathrm{mV} \mathrm{y}+138 \mathrm{mV}$ ). En estas condiciones, la capacidad de retener $\mathrm{P}$ en el sedimento no resulta favorable. Esto sugiere que las sustancias orgánicas reductoras, más que las parejas óxido-reductoras tales como $\mathrm{Fe}^{2+}$ / $\mathrm{Fe}^{3+}$, dominan a bajos ORP del sedimento, y disminuyen la capacidad de adsorción de $\mathrm{P}(\mathrm{Li}$ et al. 2007). De esta manera, si hubiera periodos de liberación de $\mathrm{P}$ desde los sedimentos, los sitios de fijación quedarían alternativamente con mayor capacidad de fijación.

Los bajos porcentajes de fijación de $\mathrm{P}$ indican que el sedimento, en particular en el centro de la bahía, estaría saturado en su capacidad para retener fósforo. Larsen et al. (1981), Böstrom et al. (1982), Golterman et al. (1983) y Håkanson 
\& Jansson (1983) mostraron que en ambientes ricos en nutrientes, la liberación de $\mathrm{P}$ desde los sedimentos puede exceder los ingresos externos de fósforo ("carga interna"). En consecuencia, ocurre un flujo neto de $\mathrm{P}$ desde los sedimentos a la columna de agua. Los resultados obtenidos en este trabajo apoyan este fenómeno.

Los primeros efectos del proceso de liberación de P desde los sedimentos hacia la columna de agua se vieron reflejados en los incrementos en las concentraciones de nutrientes totales en el agua del lago y en los sitios dentro de la bahía (Temporetti et al. 2008). Este aumento de la concentración de nutrientes podría haber originado un incremento de la concentración de la clorofila a, la densidad y la biomasa del fitoplancton respecto de los muestreos previos realizados entre 2006 y 2007 (Temporetti et al. 2007). Aunque se observó un incremento en las concentraciones de nutrientes de la columna de agua, el estado trófico general del lago no se vio modificado [se mantuvo por debajo de los valores de referencia de la oligotrofia (Wetzel 2001)]. Carey \& Rydin (2011) demostraron que la distribución del fósforo total en las capas superficiales de los sedimentos se relaciona con la concentración de $\mathrm{P}$ en la columna de agua y con el estado trófico de los ambientes acuáticos. Temporetti et al. (2014) analizaron nueve testigos de sedimentos de ambientes acuáticos andino-patagónicos (incluido el Lago Lácar), y encontraron una correlación entre la concentración de PT en el sedimento y el PT de la columna de agua. Para el caso particular del Lago Lácar, estos autores hallaron que aunque el estado trófico del lago, de acuerdo a los parámetro físicos y químicos del agua, es oligotrófico, los sedimentos, en particular los de la Bahía Oriental, se comportan como sedimentos característicos de un ambiente eutrófico.

\section{CONClusiones}

Los resultados nos permiten concluir que, si bien los sedimentos de la zona de descarga del ríoPocahullopresentaron texturas gruesas (que los harían mas permeables y menos reactivos), las corrientes generadas por el ingreso del río transportan P, N y C hacia el centro de la bahía. Los sedimentos del resto de la Bahía Oriental también mostraron un deterioro debido a la acumulación de nutrientes, evidenciado por una baja capacidad de retención de P. La composición química y las experiencias de fijación/liberación indican la existencia de liberación de $\mathrm{P}$ desde los sedimentos hacia la columna de agua, sobre todo en la zona central de la bahía. Esto evidencia el proceso de carga interna. En los procesos de eutrofización de los ambientes acuáticos, esta carga interna puede llegar a ser tan importante como la carga externa; en muchos casos, esto hizo fracasar la recuperación de los ambientes afectados aun cuando se redujeran los aportes externos. La recuperación de ambientes eutrofizados es muy lenta debido a los procesos de liberación de nutrientes desde los sedimentos.

Agradecimientos: A G Beamud por la ayuda con los análisis estadísticos realizados; M Díaz, G Baffico y M Ugucioni por la ayuda en la toma y procesamiento de las muestras; C Rucchi y M Guido (Organismo de Control Municipalidad de San Martín de los Andes) por su colaboración en las tareas de coordinación en campo y laboratorio. Este trabajo fue financiado con fondos de la Secretaría de Políticas Universitarias, Ministerio de Educación de la Nación Argentina, proyecto N 04-02-101.

\section{BiBLIOGRAFÍA}

Anshumali, A \& L Ramanathan. 2007. Phosphorus fractionation in surficial sediments of Pandoh Lake, Lesser Himalaya, Himachal Pradesh, India. Applied Geochemistry, 22:1860-1871.

APHA. 1995. Standard Methods for the Examination of Water and Wastewater. 18th Ed. American Public Health Association.Washington. Pp. 1134.

Böstrom, B; M Jansson \& C Forsberg. 1982. Phosphorus Release from Lake sediments. Arch. Hydrobiol. Beith. Erg. Limnol., 18:5-59.

CAREY, C \& E RYDIN. 2011. Lake trophic status can be determined by the depth distribution of sediment phosphorus. Limnol. Oceanogr., 56:2051-2063.

CARPENTER, S. 2005. Eutrophication of aquatic ecosystems: Bistability and soil phosphorus. PNAS, 102:1000210005.

CARTER, M. (ED.). 1993. Soil Sampling and Methods of Analysis. Canadian Society of Soil Science. Lewis Pub. Pp. 823.

Cooke, S; P Temporetti \& F Pedrozo. 2006. Estado de Situación del lago Lácar y Arroyo Pocahullo y su Evolución Trófica. San Martín de los Andes - Pcia. del Neuquén. Informe final pasantía, Centro Regional Universitario Bariloche, Universidad Nacional del Comahue. Pp. 25.

CordinI, R. 1964. El Lago Lácar del Parque Nacional Lanín (Neuquén). Anales de Parques Nacionales, 10(2):111-150.

DE Vicente, I; L Serrano; V Amores; V Clavero \& L CruzPizarro. 2003. Sediment phosphate fractionation and interstitial water phosphate concentration in two coastal lagoons (Albúferas de Adra, SE Spain). Hydrobiologia, 492:95-105.

EINSELE, W. 1936. Uber die Beziehungen des Eisenkreislaufs zum Phosphatkreislauf im eutrophen See. Arch. Hydrobiol., 29:664-686.

EINSELE, W. 1938. Uber chemische und kolloidchemische Vorgange in Eisen-Phosphat-Systemen unter limnochemischen und limnogeologischen Gesichtspunkten. Arch. Hydrobiol., 33:361-387.

FrIEDMAN, GM; JE SANDERS; \& DC KOPASKA-MERKEL. 1992. Principles of Sedimentary Deposits. Stratigraphy and Sedimentology. Macmillal Publ. Co., New York. Pp. 717.

Golterman, HL; PG SLy \& RL Thoma. 1983. Study of 
the relationship between water quality and sediment transport. UNESCO. Pp. 231.

Golterman, HL. 2004. The Chemistry of Phosphate and Nitrogen Compounds in Sediments. Kluwer Academic Publichers, London. Pp. 280.

HÅKAnSON, L \& M Jansson. 1983. Principles of Lake Sedimentology. Springer-Verlag, Heidelberg. Pp. 316.

HÅKANSON, LH. 2003. Quantifying Burial, the Transport of Matter from the Lake Biosphere to the Geosphere. Internat. Rev. Hydrobiol., 88(5):539-560.

Hieltjes, AH \& L LIJKLEMA. 1980. Fractionation of inorganic phosphates in calcareous sediments. J. Environm. Qual., 9:405-407.

Horne, AJ \& ChR Goldman. 1994. Limnology. McGraw Hill Inc, N.Y. Pp. 576.

Kaiserli, A; D Voutsa \& C Samara. 2002. Phosphorus fractionation in lake sediments - Lakes Volvi and Koronia, N. Greece. Chemosphere, 46:1147-1155.

Katsev, S; I Tsandev; I L'Heureux \& D Rancourt. 2006. Factors controlling long-term phosphorus efflux from lake sediments: Exploratory reactive-transport modelling. Chemical Geology, 234:127-147.

Koski-VÄhäLÄ, J; H Hartikainen \& P TAllberg. 2001 Phosphorus Mobilization from Various Sediment Pool in Response to Increased $\mathrm{pH}$ and Silicate Concentration. J. Environ. Qual., 30:546-552.

Langmuir, D (ED.). 1997. Aqueous Environmental Geochemistry. Pearsson, Prentice Hall. Pp. 601.

Larsen, K; L Shults \& A MaLneg. 1981. Summer Internal Phosphorus Supplies in Shagawa Lakes, Minnesota. Limnol. Oceanogr., 26(4):740-754.

LAST, W. 2001a. Textural analysis of Lake Sediment. En: Pp. 41-81. Last and Smol (eds.): Tracking Environemental Change Using Lake Sediments. Vol. 2, Physical and Geochemical Methods. Chapter 4.

LAST, W. 2001b. Mineralogical Analysis of Lake Sediment. En: Last and Smol (eds.). Pp. 143-187: Tracking Environemental Change Using Lake Sediments. Vol. 2, Physical and Geochemical Methods. Chapter 6.

LI, QM; W ZhanG; X WANG; Y ZHOU YI; H YanG: ET AL. 2007. Phosphorus in Interstitial Water Induced by Redox Potential in Sediment of Dianchi Lake, China. Pedosphere, 17(6):739-746.

MaAsen, S; D Uhlmann \& I Roske. 2005. Sediment and pore water composition as a basis for the trophic evaluation of standing waters. Hidrobiologia, 543:55-70.

Mortimer, CH. 1941. The Exchange of Dissolved Substances Between Mud and Water in Lake. J. Ecol., 29:280-329.

Mortimer, CH. 1942. The Exchange of Dissolved Substances Between Mud and Water lakes. J. Ecol., 30:
147-201.

Murphy, J \& JP RiLey. 1962. A Modified Single Solution Method for the Determination of Phosphate in natural Waters. Analyt. Chim. Acta., 27:31-35.

OECD. 1982. Eutrophication of waters, monitoring, assessment and control. OECD, París. Pp. 120 pp.

OlsEn, SR \& FS WATANABE. 1957. A Method to determine a phosphorus adsorption maximum of soil as measured by the Langmuir isotherm. Soil Science Society Proceedings, 144-149.

SCHINDLER, R; E HeCKY; DL FindLAY; MP Station; BR PARKER; ET AL. 2008. Eutrophication of lakes cannot be controlled by reducing nitrogen input: Results of a 37-year wholeecosystem experiment. Proc. Natl. Acad. Sci. USA, 105: 11254-11258.

SINKE, JC. 1992. Phosphorus dynamics in the sediment of a eutrophic lake. Ph.D. Thesis. University of Wageningen, The Netherlands, ISBN 90-5485-036-1.

TemporetTi, P. 1998. Dinámica del Fósforo en Cuerpos de Agua con Cría Intensiva de Salmónidos. Tesis Doctoral. Centro Regional Universitario Bariloche, Universidad Nacional del Comahue. Pp. 171.

Temporetti, P \& F Pedrozo. 2000. Phosphorus Release Rates from Sediments Affected by Fishfarming. Aquaculture Research, 31:447-455.

Temporetti, P; G Baffico; F Pedrozo; M Guido \& M Díaz. 2006. Estado Trófico Actual del Lago Lácar y el Arroyo Pocahullo y sus Principales A uentes. Informe Técnico para la Municipalidad de San Martín de los Andes, Neuquén, Etapas I y II. Pp. 24.

Temporetti, P; G Baffico; F Pedrozo; M Guido \& M Díaz. 2007. Estado Trófico del Lago Lácar y el río Pocahullo y sus Principales Afluentes. Informe Técnico para la Municipalidad de San Martín de los Andes, Neuquén, Etapas I, II y III. Pp. 80.

Temporetti, P; G Baffico; M Díaz \& F Pedrozo. 2008. Estado Trófico del lago Lácar y del río Pocahullo. In uencia de la descarga de líquidos cloacales en la cubeta Oriental del lago. Informe técnico para la Municipalidad de San Martín de los Andes, Neuquén. Pp. 44.

Temporetti, P; G Beamud \& F Pedrozo. . 2014. The trophic state of Patagonian Argentinean lakes and their relationship with distribution in depth of phosphorus in sediments. International Journal of Environmental Research, 8:671-686.

WANG, S; X Jin; H ZAHO; X ZoHU \& F Wu. 2008. Effects of Organic Matter on Phosphorus Release Kineticcs in Different Throphic Lakes Sediments and Application of Transition State Theory. Journal of Environmental Management, 88:845-852.

Wetzel, R. 2001. Limnology. Academic Press, San Diego, Philadelphia. Pp. 743. 